\title{
Neonatal diabetes - same disease, same gene, different outcomes
}

\author{
Vicente Rey y Formoso', Daniela Salazar ${ }^{2}$, Sofia Ferreira ${ }^{3}$, Rita Santos Silva ${ }^{3}$, Carla Costa ${ }^{3}$, \\ Cíntia Castro Correia ${ }^{3}$
}

${ }^{1}$ Pediatrics Department, Maternal and Paediatrics Centre, São João University and Hospital Centre, Porto, Portugal ${ }^{2}$ Department of Endocrinology, Diabetes, and Metabolism, São João University and Hospital Centre, Porto, Portugal

${ }^{3}$ Pediatric Endocrinology Unit, Paediatrics Department, Maternal and Paediatrics Centre, São João University and Hospital Centre, Porto, Portugal

Key words: neonatal diabetes mellitus; NDM; permanent NDM; transient NDM

Neonatal diabetes mellitus (NDM) is a rare disease [1-4], occurring in approximately 1 in 100,000-200,000 live births [1]. The condition may be transient or permanent [1-5] and is most often caused by monogenic defects [2-4].

KCNJ11 gene mutations represent the most common cause of permanent NDM (PNDM) [2,5] but are only rarely implicated in transient NDM (TNDM) [2]; the underlying mechanism differentiating these phenotypes is still unclear [1].

While its use is still considered off-label in the paediatric population $[1,4,5]$, sulfonylureas have become the mainstay of treatment for patients with KCNJ11-related NDM [1-5].

We herein present two case reports of patients with overlapping early presenting conditions, which, although they carried the same mutated gene, had acutely different outcomes.

Patient no. 1, a three-month-old female infant, with no relevant priors, was evaluated in a neighbouring hospital for respiratory distress and irritability in the previous 24 hours. She was hypotensive and dyspnoeic, and blood tests reflected dehydration and showed severe hyperglycaemia and elevated ketone bodies, for which diabetic ketoacidosis (DKA) was suspected. Intravenous (IV) hydration and insulin perfusion were started, and she was transferred to our hospital's Paediatric Intensive Care Unit (PICU).

The patient's condition improved progressively under IV insulin perfusion, acidosis resolved, and both glycaemia and ketonaemia normalised.
Blood testing revealed negative anti-insulin and anti-GAD antibodies, and an ultrasound confirmed a macroscopically normal pancreas. Due to the patient's age and clinical presentation, a genetic study for possible causes of NDM was requested.

After five days in the PICU, the patient was switched to subcutaneous insulin therapy and was subsequently transferred to our paediatrics general ward. After adequate glycaemic control was attained under a basal-bolus scheme, she was discharged (day 26).

After discharge, despite being under the same subcutaneous insulin scheme, blood glucose levels remained erratic $\left(\mathrm{HbA}_{1 \mathrm{c}} 8.5-11.4 \%\right)$. At nine months, genetic study results revealing a KCNJ11 mutation — c.602G >A (p.Arg201His) were received, confirming the diagnosis of NDM. After approval from responsible entities, the switch to off-label therapy with glibenclamide was successfully performed in an inpatient setting.

Afterwards, during the six years of follow-up in our institution, the patient was managed successfully under exclusive sulfonylurea treatment $\left(\mathrm{HbA}_{1 \mathrm{c}} 5.4-7 \%\right)$ - hypoglycaemic episodes were rare and no further insulin therapy was necessary.

Patient no. 2, a two-month-old male infant, with no relevant medical history, was assisted in another local hospital for refusal to feed, vomiting, and apparent respiratory distress. On exam, the infant was ill-appearing, dyspnoeic, and had a prolonged capillary perfusion time. Initial blood testing showed metabolic acidosis with ketonaemia and blood glucose levels over 
$500 \mathrm{mg} / \mathrm{dL}$. Intravenous hydration was initiated, and the patient was brought to our institution's PICU.

On admission, after initial assessment and review of the previous blood test results, DKA was diagnosed and IV insulin perfusion was started.

Both anti-GAD and anti-insulin antibody screenings were negative, and ultrasound revealed an apparently normal pancreas. A genetic study for NDM-associated mutations was requested.

The patient progressively improved, initially under IV insulin infusion, and then from day 5 (when he was transferred to our General Paediatrics Ward) under continuous subcutaneous insulin infusion (CSII). He was discharged on day 13, after glycaemic stability was attained and the parents were familiar with the system.

After discharge, the patient remained euglycaemic under CSII but needed progressively lower insulin dosages until, at five months of age, insulin therapy was suspended definitively.

At eight months of age genetic testing results were obtained, detecting a KCNJ11 mutation - c.149G > A (p.Arg50Gln).

He is currently three years old and remains normoglycaemic.

Neonatal diabetes mellitus most often presents within the first six months of life, frequently as DKA [4, 5], as was the case with our two patients. Nonetheless, this condition should still be considered in patients up to 12 months of age, and its course may be insidious or even subclinical at onset [4].

When evaluating infants with DKA or even apparently persistent hyperglycaemia, a high level of suspicion toward NDM is of the upmost importance, and the threshold for requesting genetic testing should be low [3-5] - genetic diagnosis may not only confirm suspicions, but also directly impact management strategies, as with the use of sulfonylureas in patients with KCNJ11 mutations [1, 3-5].

As reflected by our report, despite being otherwise useful, the detection of a KCNJ11 mutation does not accurately predict the patient's outcome because, although mostly associated with PNDM, this mutation may also cause transient forms of the condition.

Infancy-onset diabetes should prompt early genetic testing for NDM [3-5]. Close monitoring of NDM patients who carry a KCNJ11 mutation is crucial, and the possibility of a transient form should always be considered.

\section{Conflicts of interest and funding}

The authors declare there are no conflicts of interest. The authors received no specific funding for this work.

\section{References}

1. Ashcroft FM, Puljung MC, Vedovato N. Neonatal Diabetes and the K Channel: From Mutation to Therapy. Trends Endocrinol Metab. 2017; 28(5): 377-387, doi: 10.1016/j.tem.2017.02.003, indexed in Pubmed: 28262438 .

2. Barbetti F, D'Annunzio G. Genetic causes and treatment of neonatal diabetes and early childhood diabetes. Best Pract Res Clin Endocrinol Metab. 2018; 32(4): 575-591, doi: 10.1016/j.beem.2018.06.008, indexed in Pubmed: 30086875

3. De Franco E, Ellard S. Genome, Exome, and Targeted Next-Generation Sequencing in Neonatal Diabetes. Pediatr Clin North Am. 2015; 62(4): 1037-1053, doi: 10.1016/j.pcl.2015.04.012, indexed in Pubmed: 26210631.

4. Lemelman MB, Letourneau L, Greeley SA. Neonatal Diabetes Mellitus: An Update on Diagnosis and Management. Clin Perinatol. 2018; 45(1): 41-59, doi: 10.1016/j.clp.2017.10.006, indexed in Pubmed: 29406006.

5. Letourneau LR, Greeley SA. Precision Medicine: Long-Term Treatment with Sulfonylureas in Patients with Neonatal Diabetes Due to KCNJ11 Mutations. Curr Diab Rep. 2019; 19(8): 52, doi: 10.1007/s11892-019-1175-9, indexed in Pubmed: 31250216. 